\title{
Effect of international sensitivity index (ISI) of thromboplastins on precision of international normalised ratios (INR)
}

\author{
D A TABERNER, L POLLER, J M THOMSON, K V DARBY The United Kingdom Reference \\ Laboratory for Anticoagulant Reagents and Control, Withington Hospital, Manchester
}

SUMMARY The contribution of the thromboplastin international sensitivity index (ISI) to the interlaboratory coefficient of variation (CV) of the international normalised ratio (INR) with individual reagents was assessed. In theory the precision of the INR should increase with lower ISI values. An empirical relation has been established between the ISI, the INR, and its CV for two rabbit thromboplastins used in sufficient numbers for analysis in the United Kingdom. This was based on the cumulative data from the United Kingdom National External Quality Assessment Scheme (NEQAS) surveys over two years beginning in 1986. The actual precision achieved in NEQAS for the two reagents depends on the ISI value of the thromboplastin and it agreed closely with the figure predicted by the empirical model.

The findings show that the ISI value of a thromboplastin strongly influences the interlaboratory variability of the INR obtained with it. The CV of the INR approximates to the CV of the prothrombin ratio multiplied by the ISI. Manufacturers of thromboplastin should therefore be encouraged to produce reagents showing good precision of results reported as simple prothrombin ratios and a low ISI value to avoid impairment in precision when ratio results are transformed to INR.

A revised international procedure for standardisation of the prothrombin time (PT) in anticoagulant control was accepted by the international committees ${ }^{1}$ and approved by the World Health Organization. ${ }^{2}$ It is based on reporting PT results as international normalised ratios (INR) derived from an international sensitivity index (ISI) for an individual thromboplastin reagent. The latter quantifies responsiveness of a PT reagent to the coumarin induced defect. The ISI value is obtained from calibration of the individual reagent against an international reference preparation for thromboplastin. A patient's prothrombin ratio is then converted into an INR by the formula:

$$
\text { INR }=\text { (prothrombin ratio })^{\mathbf{I S I}}
$$

Any lack of precision in determining the local prothrombin ratio will thus be carried over and transformed in the conversion to the INR.

The influence of the ISI value on the precision of the INR with a variety of Quick test reagents is considered in this study. Analysis was undertaken from both the theoretical standpoint and from the actual results observed in cumulative PT exercises in the United

Accepted for publication 4 August 1988
Kingdom National External Quality Assessment (NEQAS) in blood coagulation between 1980 and 1988.

\section{Material and methods}

Analysis of performance in PT testing has been based on NEQAS surveys conducted at over $\mathbf{4 0 0}$ centres in the United Kingdom, at intervals of three months. A PT exercise was included in each of the serial surveys. This incorporated two or three test samples obtained from single donations from patients stabilised on long term treatment with coumarin. Persistent poor performance (PPP) in the PT has been defined as a deviation of $15 \%$ or more from the reagent mean in two consecutive or three of five serial exercises. Data on the numbers of PPP have been available since 1982 following the definition of the criteria by the NEQAS haematology panel. The incidence of PPP in the prothrombin time test in the NEQAS exercises was analysed with reference to the thromboplastin used and related to its ISI.

In a recent report the relation between the mean prothrombin ratio and its standard deviation (SD) in NEQAS exercises was found to be linear when plotted 
on a logarithmic scale. ${ }^{3}$ The slope and intercept in this relation were determined from NEQAS prothrombin returns between 1980-1987, during which the predominant thromboplastin, Manchester Comparative Reagent (MCR), was of human brain origin. Since 1986 rabbit tissue thromboplastins have been used instead and these may not show the same relation between precision and prothrombin ratio (PR) as those found with human brain MCR. The relation between prothrombin ratio and SD obtained in the eight subsequent NEQAS prothrombin exercises in 1986 and 1987 was therefore determined for the two rabbit tissue thromboplastins (Manchester Reagent (MR) and Diagen A (DA)) used in sufficient numbers for analysis. In the surveys, results from participants were expressed as prothrombin time, not prothrombin ratio. In this study the PT results were interpreted as mean ratios based on the mean stated normal values with the respective reagents. The slope and intercept parameters in the relations were calculated. Using these parameters, empirical relations between the prothrombin ratio and its $\mathrm{CV}$ were established for these two thromboplastins and compared with that determined for MCR in the previous survey of PT exercises in NEQAS. ${ }^{3}$

As the INR is the prothrombin ratio raised to the power of the ISI, then intrinsically the CV of the INR depends on the ISI as well as on the CV of the prothrombin ratio. If

$$
Y=f(x)
$$

then by the method of differentials:

It then follows directly that:

$$
\operatorname{SD}(Y)=\operatorname{SD}(X) \cdot f^{\prime}(x)
$$

$$
\text { SD(INR) = ISI.PR }{ }^{\text {ISI-1 }} . S D(P R)
$$

and SD INR/PR ${ }^{i \mathrm{SII}}=$ ISI.SD(PR)/PR

which gives $\mathrm{CV}(\mathrm{INR}) \bumpeq \mathrm{ISI} \mathrm{CV}(\mathrm{PR})$

for ISI with values between 1.0-2.6 and CV (PR) between $5 \%$ and $15 \%$ - that is, the CV of the INR is the product of the ISI and the CV of the prothrombin ratio.

The empirical graphical relation between the prothrombin ratio and its $\mathrm{CV}$ was therefore modified using the above formula to determine the relation between the INR and its CV. The mean INR results for the 20 plasmas included in the eight NEQAS surveys and mean CV (INR) obtained were compared with the graphical relation between the INR and its CV obtained with the two rabbit thromboplastins - that is, MR and DA. This was undertaken to test the theory that the CV of the INR approximates to the $\mathrm{CV}$ of the prothrombin ratio multiplied by the ISI.

\section{Results}

The incidence of poor performance increased considerably after the withdrawal of the human brain reagent MCR (fig 1). Fig 2 shows that the increase is due to relatively poor precision with reagents of ISI values of 1.4 or more.

The relation between the logarithms of the SD and the PR was linear for the respective thromboplastins, although the slope and intercepts differed (fig 3).

The relation between the prothrombin ratio and its CV (fig 4) was steeper for the reagent with an ISI of 1.4 (DA) than with the reagent with a low ISI of $1 \cdot 1$ (MR) and similar to that obtained from our previous study. ${ }^{4}$ The latter was based predominantly on data for the human brain MCR results (ISI 1.0). In contrast, the slope for MR is less steep than that for MCR.

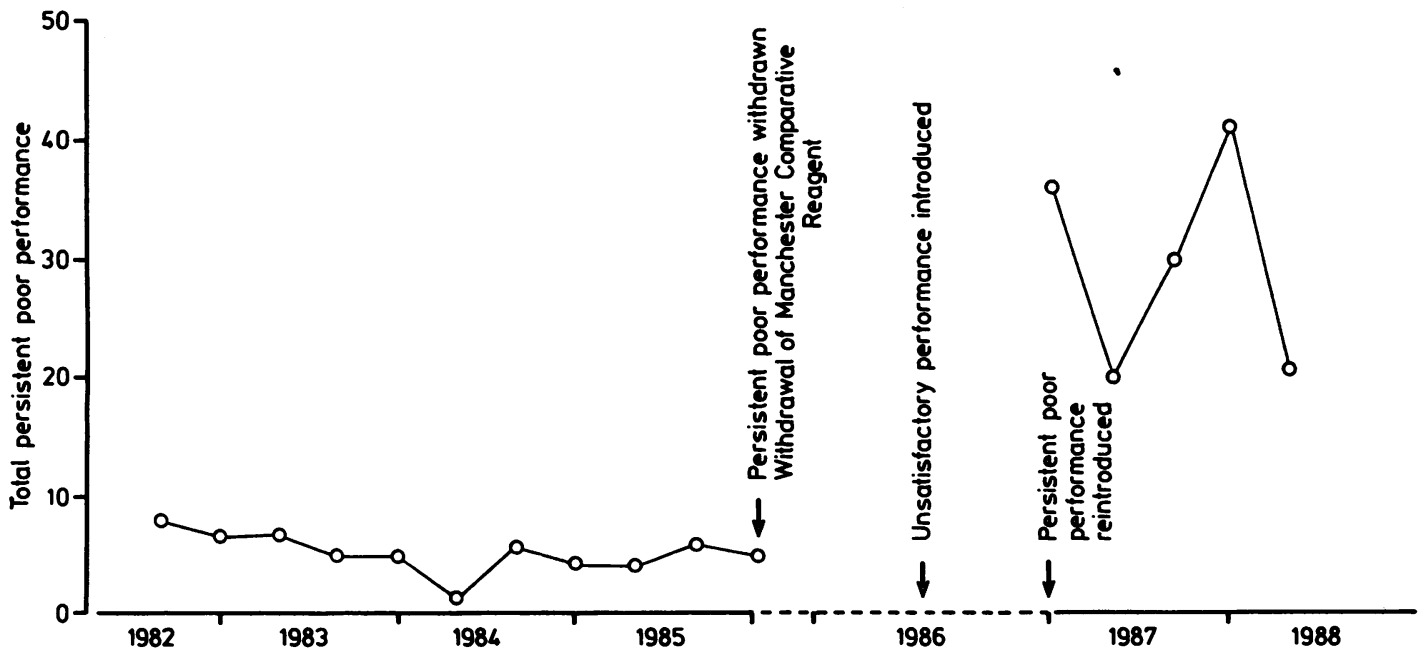

Fig 1 Incidence of persistent poor performance in PT in serial NEQAS surveys (1982-1988). 
94

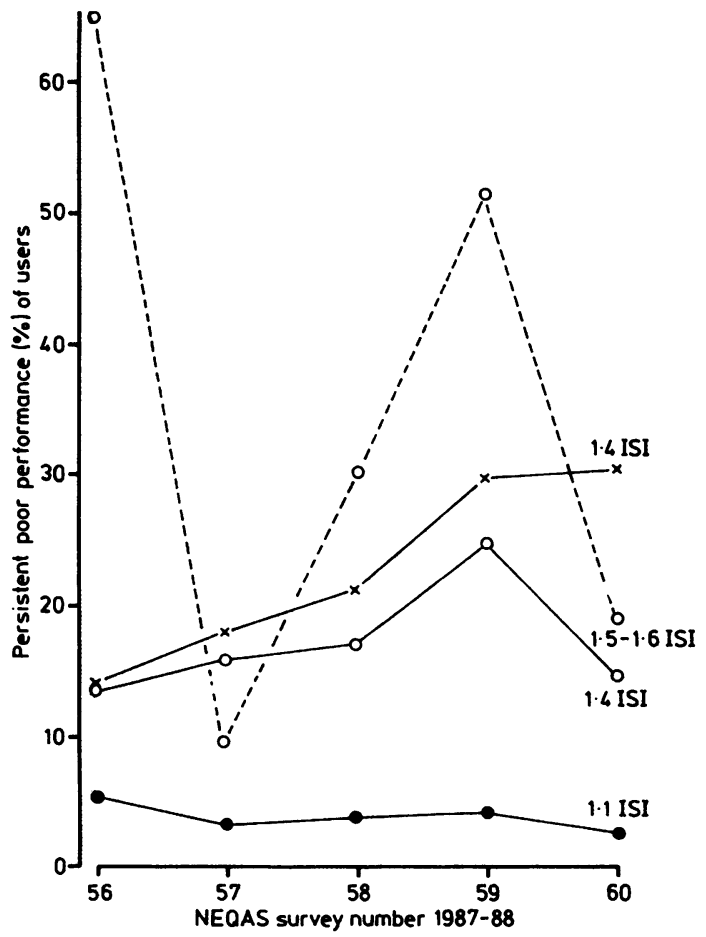

Fig 2 Incidence of persistent poor performance in $P T$ according to rabbit thromboplastin reagent used and ISI.
Taberner, Poller, Thomson, Darby

The transformation of prothrombin ratio to INR increases its $\mathrm{CV}$ by a factor which is approximately the product of the ISI:

$$
\mathrm{CV}(\mathrm{INR})=\mathrm{CV}(\mathrm{PR}) \times \mathrm{ISI}
$$

The relation between CV(INR) and INR is shown in fig 5 , based on the empirical formulae derived from NEQAS data in fig 4 and the approximate theoretical relation:

$$
\mathrm{CV}(\mathrm{INR})=\mathrm{CV}(\mathrm{PR}) \times \mathrm{ISI}
$$

Transformation to INR increased its CV but exaggerated the difference between the slopes of the low and the higher ISI rabbit brain reagents. The mean INR obtained in the 1986 and 1987 exercises for the two rabbit thromboplastins with sufficient numbers for analysis and their respective $\mathrm{CV}$ are also plotted in fig 5 and closely agreed with the $\mathrm{CV}$ predicted from the mean INR using the statistical model.

\section{Discussion}

Following the introduction of the widespread use of rabbit tissue thromboplastin reagents in the United Kingdom in 1986, it has become apparent that precision in the United Kingdom NEQAS exercises for PT has deteriorated considerably. ${ }^{4}$ The present study shows that the incidence of persistent poor performance also increased and was greater with reagents with higher ISI values. Nevertheless, the performance with the rabbit brain reagent with a low ISI value $(1 \cdot 1)$

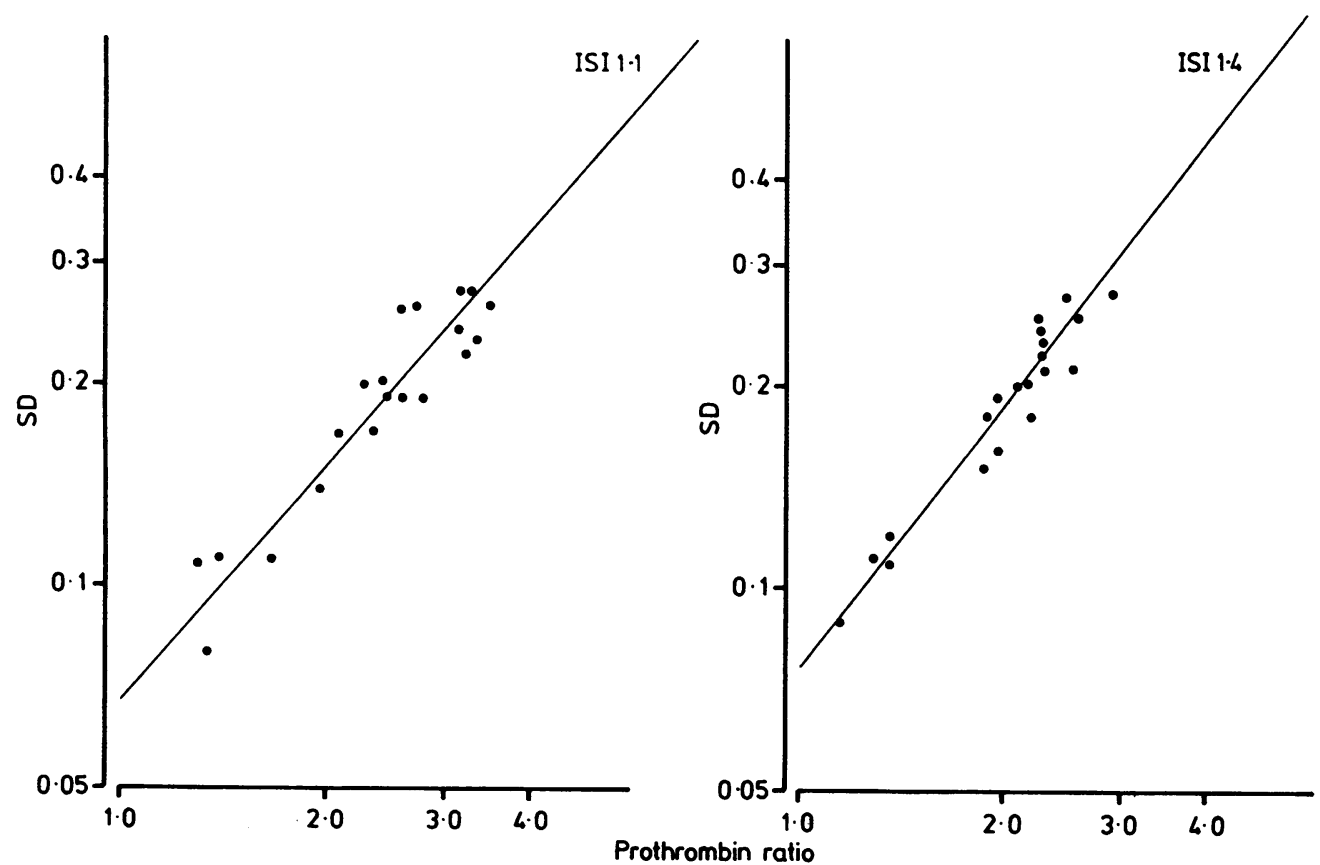

Fig 3 Linear regression plot of SD and simple prothrombin ratio for the two thromboplastins. 


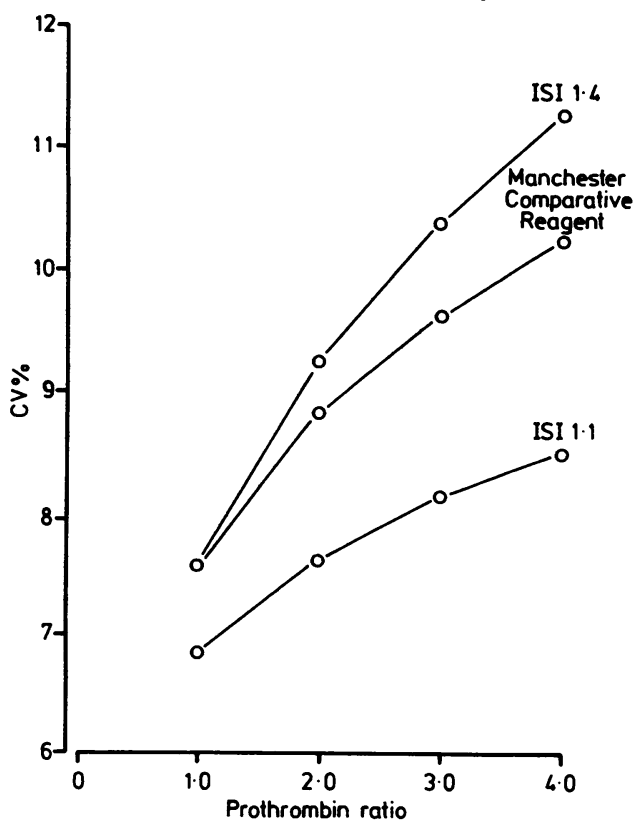

Fig 4 Empirical relation between $C V$ and simple prothrombin ratio.

has remained close to that previously achieved with the human brain reagent MCR. The present study shows that when two reagents with ISI values of $1 \cdot 1$ and 1.4 are compared, the precision of the INR depends on two factors.

The first component is the precision of the simple prothrombin ratio. Analysis of NEQAS data obtained during 1986 and 1987 with 20 lyophilised single donations of plasma coumarin shows that the CV of prothrombin ratio is described by a power relation (fig 4).

Plots of the power relations for the three thromboplastins with an ISI of $1 \cdot 4,1 \cdot 1$, and 1.0 respectively, with the constants determined from NEQAS data, show that the first has a much steeper slope than the low ISI rabbit preparation and is slightly steeper than human brain MCR. The precision in ratio terms is greater with the rabbit reagent with the low ISI $(1 \cdot 1)$ than with the human brain reagent MCR (ISI 1.0). In contrast, the precision in ratio terms is closer to the higher ISI rabbit reagent (1.4) to that with the human brain reagent MCR (fig 4).

The second component determining precision of the INR of a thromboplastin is its ISI value. This study has shown that the CV of INR is about equal to the coefficient of variation of the prothrombin ratio multiplied by the ISI. The reduction in precision in the CV(INR) is therefore more pronounced for the thromboplastin with an ISI of 1.4 than that with an ISI of

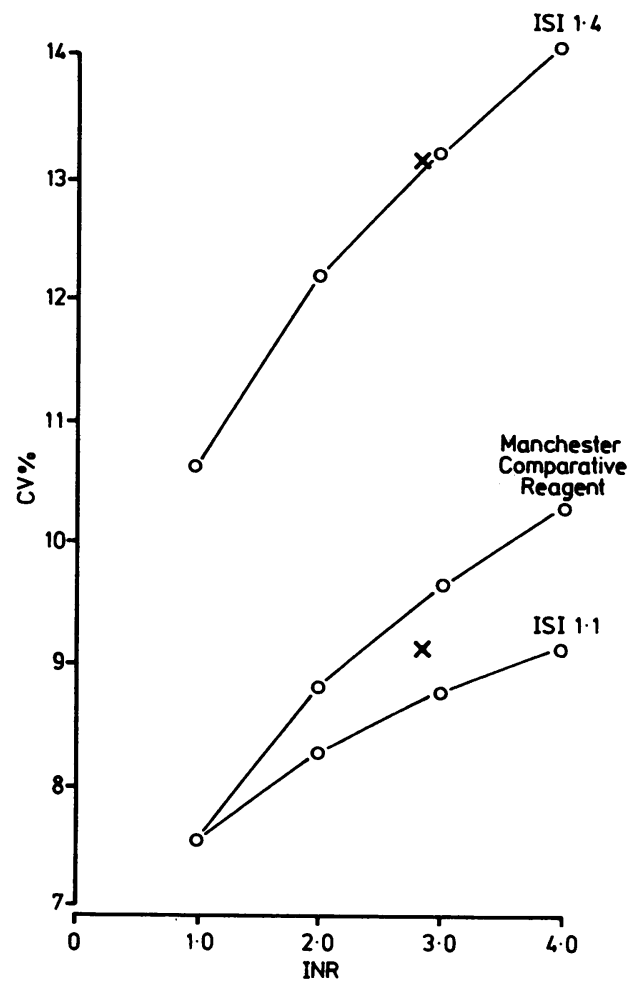

Fig 5 Empirical relation between $C V$ and INR. Upper cross represents mean $C V$ and mean INR obtained in NEQAS surveys with a thromboplastin and an ISI of 1.4 (DA). Lower cross shows similar results for a thromboplastin with an ISI of $1 \cdot 1(M R)$.

$1 \cdot 1$. The relation between the INR and its $\mathrm{CV}$ is similar for MR and MCR (fig 5), and much less steep than with the higher ISI rabbit thromboplastin.

The actual results obtained from NEQAS surveys for 1986 and 1987 show that the mean INR and mean CV of the INR results for the two rabbit brain thromboplastins closely approximated to that predicted by the empirical model (fig 5). A considerable part of the deterioration in precision reported in NEQAS is therefore due to the effect of the higher ISI thromboplastins. It may seem paradoxical that the MR reagent with an ISI of $1 \cdot 1$ gave greater precision than MCR $($ ISI $=1.0)$. This is based on the greater precision of the prothrombin ratio with MR, which, when multiplied by the ISI, still gives a lower CV of the INR than the human brain reagent MCR. Therefore, this does not invalidate the principle of the effect of the ISI but shows the importance of good precision of the PTs with individual thromboplastin reagents.

The results of this study indicate that in theory the ISI strongly influences the interlaboratory variability of the INR obtained, as the CV of the INR approx- 
imates to the $\mathrm{CV}$ of the prothrombin ratio multiplied by the ISI. Recent NEQAS results with the two reagents used in sufficient numbers for analysis are entirely in keeping with this approximation, the CV with the thromboplastin of ISI 1.4 being $14 \%$ for an INR of 4.0 , while that of the lower ISI reagent ranged between $9-10 \%$ for the same INR. Overall precision in PT testing with a reagent is affected by factors other than the ISI. For instance, the stability of the reagent, interbatch variation, and methods of end point detection may have a direct influence. It has been shown in a separate study ${ }^{4}$ that the precision of testing with DA reagent was improved by the use of the $\mathrm{KC} 4 / 10$ coagulometer system. Other reagents or test systems may have greater or less effects on precision of the prothrombin ratio result. Nevertheless, the ISI must, on the present evidence, be regarded as an important influence on the precision of the INR.

\section{References}

1 Loeliger EA, Lewis SM. Progress in laboratory control of anticoagulants. Lancet ii:318-20.

2 World Health Organization. Expert Committee on Biological Standardisation. 33rd Report. WHO Technical Report Series. Geneva: WHO, 1983:1-105.

3 Poller L, Taberner DA, Thomson JM, Darby KV. A survey of prothrombin time results in NEQAS exercises 1980-1987. J Clin Pathol 1988;41:361-4.

4 Poller L, Thomson JM, Taberner DA. Effect of automation on prothrombin time test in NEQAS surveys. J Clin Pathol. 1989;42:99-100.

Requests for reprints to: Dr L Poller, UK Reference Laboratory for Anticoagulant Reagents and Control, Withington Hospital, Manchester M20 8LR, England. 